International Journal of Modern Physics A

(C) World Scientific Publishing Company

\title{
Linear and Non Linear Effects on the Newtonian Gravitational Constant as deduced from the Torsion Balance
}

\author{
M. Rossi \\ Dipartimento di Matematica, \\ Università degli Studi di Torino \\ Via Carlo Alberto 10, \\ 10123 Torino, Italy \\ L. Zaninetti \\ Dipartimento di Fisica Generale, \\ Università degli Studi di Torino \\ Via Pietro Giuria 1, \\ I-10125 Torino, Italy \\ phone +390116707460, fax +390116699579 \\ Received Day Month Year \\ Revised Day Month Year
}

\begin{abstract}
The Newtonian gravitational constant has still 150 parts per million of uncertainty. This paper examines the linear and nonlinear equations governing the rotational dynamics of the torsion gravitational balance. A nonlinear effect modifying the oscillation period of the torsion gravitational balance is carefully explored.

Keywords: Experimental studies of gravity ; Determination of fundamental constants;

PACS numbers: 04.80.-y ; 06.20.Jr;
\end{abstract}

\section{Introduction}

After many years of measurements, begun by H. Cavendish, the Newtonian gravitational constant value, said $G$, is still affected by a large error 1]. The CODATA recommends $G=(6.6742 \pm 0.001) \times 10^{-11} \frac{\mathrm{m}^{3}}{\mathrm{kgs}^{2}}$, meaning a relative standard uncertainty of 150 parts per million (in the following ppm) 2 . This value has had recent confirmation by means, on one hand, of a super-conducting gravimeter 3 and, on the other hand, of a careful analysis of the possible beam balance nonlinearity 4 . The original Cavendish method of measure, employing the torsion balance, still reveals a large discrepancy from the recommended value: about $500 \mathrm{ppm}$. This is probably due to imperfections of the crystalline structure of the torsion fibre $617 / 8$. Moreover recent studies point out that the period of a torsion pendulum might vary under disturbances of environmental noise factors, see see 9 . Other authors suggest a possible deviation from Newton's law specified as an additional contribution of 


\section{Torsion Balance}

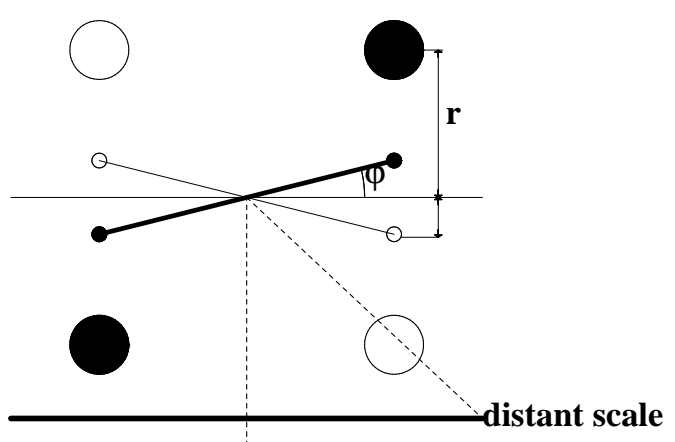

luminous source

Fig. 1. Schematic view of the torsional balance

Yukawa potential type 10. This paper first analyses the linear and non linear equations governing the torsional balance rotational dynamics (Section 2). By means of a gravitational torsion balance, same values of $G$ are obtained and summarised (Section 3). The oscillation period's variation, due to a non linear effect, is then discussed (Section 4).

\section{The basic equations}

The form of Newton's law of gravitation is

$$
F=G \frac{m M}{r^{2}},
$$

where $G$ is the gravitational constant, $M$ the great mass, $m$ the small mass and $r$ their relative distance. The Leybold balance represents a widespread instrument to determine the constant $G$, see Figure 1 and is constructed with the following components.

(1) A freely oscillating horizontal bar, of length $2 d$, holding two small lead balls of mass $m$ as in Figure 1 supported by a torsion fibre that has a torsional constant $\tau$.

(2) Two larger balls of mass $M$ that can be positioned next to the small balls as in Figure 1 The center of mass of the two $m$ and $M$ are supposed to be all on a plane perpendicular to the fibre. 


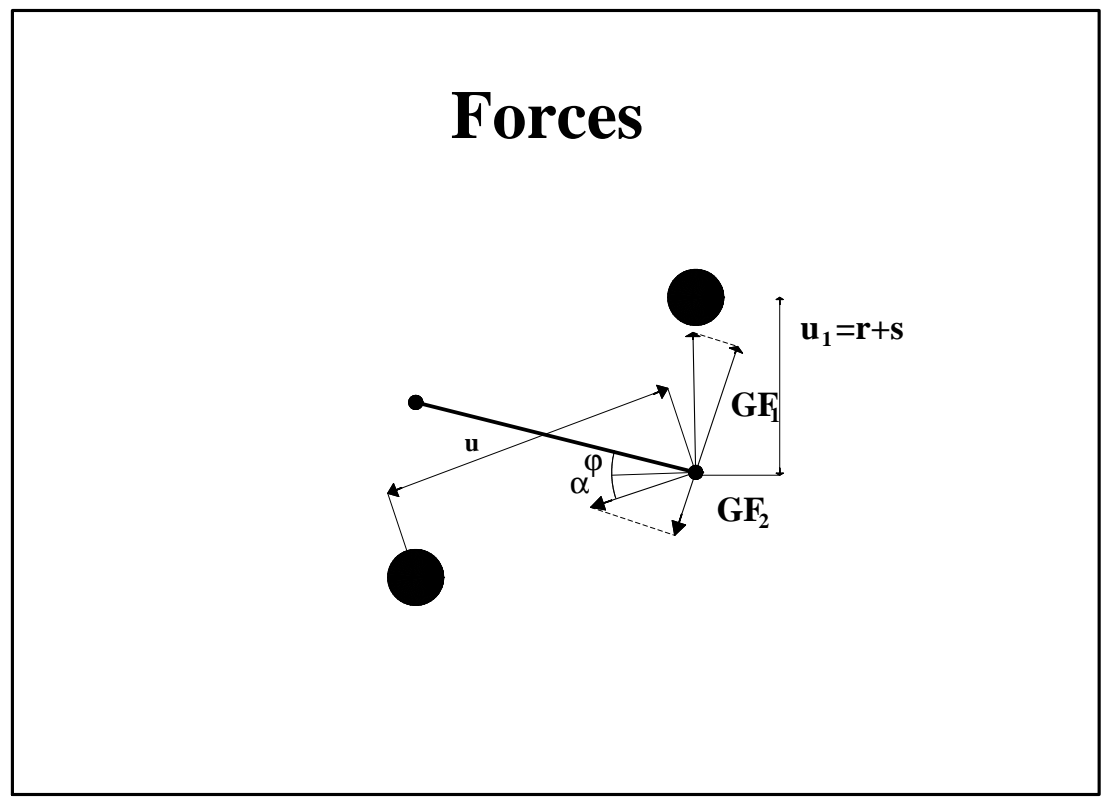

Fig. 2. Top view of the Cavendish balance

(3) A luminous source directed toward the center of mass of the bar where is reflected by a mirror.

(4) A scale at distance $l$ where the reflected light beam is measured.

Thus the equilibrium position about which the pendulum oscillates is different for the two positions and it is this difference which we use to determine G. The Figure 2 reports a plot of the motion. The moment of inertia of the bar, $I$ is

$$
I \sim 2 m d^{2} .
$$

The fundamental equation of rotational dynamics is

$$
I \ddot{\varphi}=M_{g}+M_{v}+M_{t},
$$

where

$$
\begin{aligned}
& M_{v}=-\beta \dot{\varphi}, \\
& M_{t}=-\tau \varphi,
\end{aligned}
$$

here $\beta$ is the coefficient of viscosity of air, $\varphi$ the angle between bar and bar itself when the torque is zero. This angle is measured in the anti clockwise direction. The term $M_{g}$ represents the torque of the gravitational forces. From equation (1) , we obtain

$$
M_{g}=2 d G F(\varphi)
$$


where $F$ is a function of the angle $\varphi$. The law of dependence of $F$ with $\varphi$ is complex and will here be analysed. When the motion starts the resulting force is

$$
F(\varphi)=F_{1}(\varphi)-F_{2}(\varphi)
$$

where

$$
\begin{gathered}
F_{1}(\varphi)=m M \frac{\cos \varphi}{u_{1}^{2}}, \\
F_{2}(\varphi)=m M \frac{\cos \left(\frac{\pi}{2}-(\varphi+\alpha)\right)}{u^{2}}=m M \frac{\sin (\varphi+\alpha)}{u^{2}},
\end{gathered}
$$

now $\alpha:=\arcsin \left(\frac{r-s}{u}\right)$ (where $r$ e $s$ are defined as in Figure 1). With our data, see Section 3 the maximum angular excursion of the angle $\varphi$ is

$$
\Delta=\arcsin \left(\frac{x_{\max }-x_{\min }}{2 l}\right) .
$$

The angle $\varphi$ has a low values, see Table1, and a Taylor series expansion that keep terms to order $\varphi^{2}$ will be adopted. This means to forget quantities less than $310^{-5}$. The series representation gives

$$
u_{1}=\sqrt{(r+s)^{2}+(d-d \cos \varphi)^{2}} \sim r\left(1+\varphi\left(\frac{2 d}{r}+\frac{d^{2}}{r^{2}} \varphi+\frac{d^{2}}{4 r^{2}} \varphi^{3}\right)\right)^{\frac{1}{2}} .
$$

Developing the last term with a Maclaurin series we obtain

$$
u_{1} \sim r\left(1-\frac{\varphi}{2}\left(\frac{2 d}{r}+\frac{d^{2}}{r^{2}} \varphi\right)-\frac{d^{2}}{2 r^{2}} \varphi^{2}+O\left(\varphi^{3}\right)\right) \sim r+d \varphi .
$$

As a consequence

$$
F_{1} \sim m M \frac{1-\frac{\varphi^{2}}{2}}{(r+d \varphi)^{2}}
$$

and expanding the denominator we obtain

$$
(r+d \varphi)^{-2}=\left(r^{-2}-2 \frac{d}{r^{3}} \varphi+3 \frac{d^{2}}{r^{4}} \varphi^{2}+O\left(\varphi^{3}\right)\right)
$$

that means

$$
F_{1} \sim \frac{m M}{r^{2}}\left(1-\frac{2 d}{r} \varphi+\left(\frac{3 d^{2}}{r^{2}}-\frac{1}{2}\right) \varphi^{2}\right) .
$$

An expression for $F_{2}$ can be obtained from equation (9)

$$
F_{2}=m M \frac{\sin \varphi \cos \alpha+\cos \varphi \sin \alpha}{u^{2}} \sim m M \frac{r+d \varphi-\frac{r}{2} \varphi^{2}}{\left(d^{2}+r^{2}-2 r d \varphi-d^{2} \varphi^{2}\right)^{\frac{3}{2}}} .
$$

On Taylor expanding the denominator

$$
\left(4 d^{2}+r^{2}-2 r d \varphi-d^{2} \varphi^{2}\right)^{-\frac{3}{2}}=\left(4 d^{2}+r^{2}\right)^{-\frac{3}{2}}\left(1+\frac{3 d r}{4 d^{2}+r^{2}} \varphi+\frac{3 d^{2}\left(4 d^{2}+5 r^{2}\right)}{2\left(4 d^{2}+r^{2}\right)^{2}} \varphi^{2}+O\left(\varphi^{3}\right)\right),
$$


and therefore

$$
F_{2} \sim \frac{r+\frac{4 d\left(d^{2}+r^{2}\right)}{4 d^{2}+r^{2}} \varphi+\frac{r\left(20 d^{4}+13 d^{2} r^{2}-r^{4}\right)}{2\left(4 d^{2}+r^{2}\right)^{2}} \varphi^{2}}{\left(4 d^{2}+r^{2}\right)^{\frac{3}{2}}} .
$$

Now $F$ from equation (7) can be expressed like a Taylor expansion truncated at $O\left(\varphi^{3}\right)$

$$
F \sim A_{0}+A_{1} \varphi+A_{2} \varphi^{2}
$$

where

$$
\begin{gathered}
A_{0}:=m M\left(\frac{1}{r^{2}}-\frac{r}{\left(4 d^{2}+r^{2}\right)^{\frac{3}{2}}}\right), \\
A_{1}=-2 m M\left(\frac{d}{r^{3}}+\frac{2 d\left(d^{2}+r^{2}\right)}{\left(4 d^{2}+r^{2}\right)^{\frac{5}{2}}}\right), \\
A_{2}=3 \frac{m M d^{2}}{r^{4}}-1 / 2 \frac{m M}{r^{2}}+1 / 2 \frac{m M r\left(4 d^{4}-10 r^{2} d^{2}+r^{4}\right)}{\left(4 d^{2}+r^{2}\right)^{7 / 2}} .
\end{gathered}
$$

Three methods that allow to obtain an expression for $G$ in terms of measurable quantities are now introduced. Further on the well known formula for $\mathrm{G}$ extracted from the Leybold manual is reviewed.

\subsection{Averaged $G$}

Let $F(\varphi)=\bar{F}$ for all the experience ; in first approximation we may assume that $F$ is given by equation (19) to first order

$$
\bar{F}=A_{0}+A_{1} \bar{\varphi},
$$

where $\bar{\varphi}$ is the average of the values that $\varphi$ assumes between the first position, $P_{1}$, and the last position , $P_{\infty}$, of the balance ; $A_{0}, A_{1}$ are given by equations ( 201) and (21) . The differential equation that describes the motion is

$$
I \ddot{\varphi}+\beta \dot{\varphi}+\tau \varphi=2 d G \bar{F}
$$

and it's solution is

$$
\varphi(t)=c e^{-\delta t} \cos (\omega t+\phi)+\frac{2 d G \bar{F}}{\tau},
$$

where $c$ represents the amplitude and

$$
\begin{gathered}
\delta:=-\frac{\beta}{2 I}, \\
\omega:=\frac{\sqrt{4 I \tau-\beta^{2}}}{2 I} .
\end{gathered}
$$


The angle $\varphi_{\infty}$, that represents the bar position at the end of the phenomena $P_{\infty}$ can be determined as follows

$$
\varphi_{\infty} \sim \frac{x_{\infty}-x^{o}}{2 l}=\frac{x_{\infty}-x_{1}}{4 l}
$$

and should be the same as predicted by the theory

$$
\lim _{t \rightarrow+\infty} \varphi(t)=\frac{2 d G \bar{F}}{\tau}
$$

therefore

$$
G=\frac{\tau \varphi_{\infty}}{2 d \bar{F}}
$$

In order to continue a value for $\tau$ should be derived. This can be obtained from the period of oscillation of the bar

$$
T=\frac{2 \pi}{\omega}=\frac{4 \pi I}{\sqrt{4 I \tau-\beta^{2}}}
$$

We continue by identifying $T$ with the empirical value $\bar{T}$. We continue on assuming that $\frac{\beta^{2}}{4 I}$ is small ; therefore from equations (22), (30) and (23), the following is obtained

$$
G=\frac{2 \pi^{2} I \varphi_{\infty}}{d\left(A_{0}+A_{1} \bar{\varphi}\right) \bar{T}^{2}}
$$

\section{2. $G$ with air viscosity}

From formula (44) is possible to deduce the viscosity of the air once the coefficient of damping $\delta$ is known, see Section 3 on data analysis. From formula (30) and (26) we should add to the value of $G$ reported in equation (32)

$$
G_{\beta}:=\frac{\beta^{2} \varphi_{\infty}}{8 I d \bar{F}}
$$

obtaining

$$
G=\frac{2 \pi^{2} I \varphi_{\infty}}{d\left(A_{0}+A_{1} \bar{\varphi}\right) \bar{T}^{2}}+\frac{\beta^{2} \varphi_{\infty}}{8 I d\left(A_{0}+A_{1} \bar{\varphi}\right)}
$$

\subsection{G to the first order}

Let assume that $F(\varphi)$ is not constant, we can assume at the order $O\left(\varphi^{2}\right)$ with the aid of formula (19)

$$
F \sim A_{0}+A_{1} \varphi,
$$

where $A_{0}$ e $A_{1}$ are defined in equations (20) and (21) respectively. In this case the law of motion is still equation (3)

$$
I \ddot{\varphi}+\beta \dot{\varphi}+\left(\tau-2 d G A_{1}\right) \varphi=2 d G A_{0} \quad,
$$


and the solution is

$$
\varphi(t)=c e^{-\delta t} \cos \left(\omega^{\prime} t+\phi\right)+\frac{2 d G A_{0}}{\tau-2 d G A_{1}},
$$

where the angular velocity $\omega^{\prime}$ has now the following expression

$$
\omega^{\prime}:=\frac{\sqrt{4 I\left(\tau-2 d G A_{1}\right)-\beta^{2}}}{2 I} .
$$

As a consequence

$$
\begin{gathered}
\tau=\frac{4 \pi^{2} I}{\bar{T}^{2}}+2 d G A_{1}+\frac{\beta^{2}}{4 I}, \\
\varphi_{\infty}=\lim _{t \rightarrow+\infty} \varphi(t)=\frac{2 d G A_{0}}{\tau-2 d G A_{1}},
\end{gathered}
$$

and therefore

$$
G=\frac{\tau \varphi_{\infty}}{2 d\left(A_{0}+A_{1} \varphi_{\infty}\right)}
$$

Once equation (39) is substituted in this relationship we obtain

$$
G=\frac{2 \pi^{2} I \varphi_{\infty}}{d A_{0} \bar{T}^{2}}+\frac{\beta^{2} \varphi_{\infty}}{8 d I A_{0}} .
$$

\subsection{G from Leybold manual}

The deduction of $\mathrm{G}$ through the Leybold torsional balance is widely known, see 11. We simply report the final expression

$$
G=\frac{\pi^{2} b^{2} d \Delta S}{M T^{2} l} \times(1+\beta)
$$

where

$$
\beta=\frac{b^{3}}{\left(b^{2}+4 d^{2}\right) \sqrt{b^{2}+4 d^{2}}} .
$$

The meaning of the symbols is

- $b$ :Distance between center of the great mass and small mass

- $\Delta \mathrm{S}$ :Total deflection of the light spot

- $d$ :The length of the lever arm

- $l$ :Distance between mirror and screen

- $M$ : Great mass

- T Period of the oscillations 


\begin{tabular}{lcl}
\hline \hline parameter & value & unit \\
\hline$M$ & $\left(1.5 \pm 10^{-3}\right)$ & $\mathrm{Kg}$ \\
$m$ & $\left(1.5 \cdot 10^{-2} \pm 10^{-3}\right)$ & $\mathrm{Kg}$ \\
$r$ & $\left(4.65 \cdot 10^{-2} \pm 10^{-3}\right)$ & $\mathrm{m}$ \\
$d$ & $\left(5.0 \cdot 10^{-2} \pm 10^{-3}\right)$ & $\mathrm{m}$ \\
$l$ & $\left(5.475 \pm 10^{-3}\right)$ & $\mathrm{m}$ \\
$\varphi$ & $\left(-6.715 \cdot 10^{-3} \pm 1.3 \cdot 10^{-5}\right)$ & $\mathrm{rad}$ \\
$\beta$ & $\left(1.432 \cdot 10^{-7} \pm 1.11 \cdot 10^{-8}\right)$ & $\frac{\mathrm{kg} \cdot \mathrm{m}^{2}}{\mathrm{~s}}$ \\
\hline \hline
\end{tabular}

\begin{tabular}{lcl}
\hline \hline parameter & value & unit \\
\hline$A_{0}$ & $\left(8.188 \cdot 10^{-2} \pm 1.11 \cdot 10^{-4}\right)$ & $m$ \\
$A_{1}$ & $\left(0.1470 \pm 2.85 \cdot 10^{-4}\right)$ & $m$ \\
$T$ & $(552.98 \pm 0.16)$ & $s$ \\
$\tau$ & $(1047.0 \pm 4.8)$ & $s$ \\
\hline \hline
\end{tabular}

\begin{tabular}{lllll}
\hline \hline method & equation & value & uncertainty [ppm] & accuracy \%] \\
$G$ averaged & (32) & $(6.67 \pm 0.34) \cdot 10^{-11} \frac{\mathrm{m}^{3}}{\mathrm{~kg} \cdot \mathrm{s}^{2}}$ & 52433 & 0.0161 \\
$G$ with air viscosity & $(34)$ & $(6.72 \pm 0.35) \cdot 10^{-11} \frac{\mathrm{m}^{3}}{\mathrm{~kg} \cdot \mathrm{s}^{2}}$ & 52433 & 0.69 \\
$G$ to the first order & $(42)$ & $(6.80 \pm 0.34) \cdot 10^{-11} \frac{\mathrm{m}^{3}}{\mathrm{~kg} \cdot \mathrm{s}^{2}}$ & 49989 & 1.92 \\
$G$ from Leybold manual & $(43)$ & $(6.71 \pm 0.33) \cdot 10^{-11} \frac{\mathrm{m}^{3}}{\mathrm{~kg} \cdot \mathrm{s}^{2}}$ & 49600 & 0.64 \\
\hline \hline
\end{tabular}

\section{Analysis of the data}

The physical parameters as well their uncertainties are reported in Table 1. The data were analysed through the following fitting function

$$
y(t)=A_{0}+A_{1} \cos \left(\frac{2 \pi t}{T}\right) \exp \left(-\frac{t}{\tau}\right)
$$

The data has been processed through the Levenberg-Marquardt method ( subroutine MRQMIN in 12) in order to find the parameters $A_{0}, A_{1}, T$ and $\tau$. The results are reported in Table 2 together with the derived quantities.

The value of $G$ can be derived coupling the basic parameters of the torsion balance, see Table 1, and the measured parameters of the damped oscillations, see Table 2. Table 3 reports the four values of $G$ here considered with the uncertainties expressed in absolute value and in ppm ; the precision of the measure in respect of the so called "true" value is also reported. A considerable source of error is the uncertainty in the determination of the span between the two spheres that in our case is $\approx 10^{-3} \mathrm{~m}$. Adopting a rotating gauge method 13 the uncertainty in the determination of the span between the two spheres is $\approx 0.5 \times 10^{-6} \mathrm{~m}$; this is the way to lower the uncertainty in Table 3. 


\section{Non linear effects in the vacuum}

By starting from the equation of rotational dynamics up to the second order

$$
I \ddot{\varphi}+\beta \dot{\varphi}+\left(\tau-2 d G A_{1}\right) \varphi-2 d G A_{0}=2 d G A_{2} \varphi^{2},
$$

the case of $\beta=0$ is analysed,

$$
I \ddot{\varphi}+\left(\tau-2 d G A_{1}\right) \varphi-2 d G A_{0}=2 d G A_{2} \varphi^{2},
$$

that corresponds to perform the experiment in the vacuum. On dropping the constant term and dividing by $I$ we obtain

On imposing

$$
\ddot{\varphi}+\frac{\left(\tau-2 d G A_{1}\right)}{I} \varphi=\frac{2 d G A_{2}}{I} \varphi^{2} .
$$

$$
\omega_{0}^{2}=\frac{\left(\tau-2 d G A_{1}\right)}{I},
$$

the nonlinear ordinary differential equation, in the following ODE, has the form

$$
\ddot{\varphi}+\omega_{0}^{2} \varphi=\frac{2 d G A_{2}}{I} \varphi^{2} .
$$

On adopting the transformation $T=t * \omega_{0}$ the nonlinear ODE is

$$
\ddot{\varphi}+\varphi-\epsilon \varphi^{2}=0,
$$

where

$$
\epsilon=\frac{2 d G A_{2}}{\left(\tau-2 d G A_{1}\right)} .
$$

The solution of equation (51) is reported in the Appendix Appendix A and in our case $\epsilon=0.0187$. We now have a measured period , $T_{M S}$, that is equalised to the non linear value, $T_{N L}$. The period of the linear case, $T_{L}$, can be written as

$$
T_{N L}=T_{M S}=1.00014 T_{L}
$$

and therefore

$$
T_{L}=\frac{T_{M S}}{1.00014} .
$$

In the various formulae of $G$ without damping, for example equations (32) and (43), the periods $T_{L}$ and $T_{M S}$ are raised to the square

$$
T_{L}^{2}=\frac{T_{M S}^{2}}{1.00029},
$$

and in the denominator, making the non linear $G_{N L}$ greater than the linear $G_{L}$

$$
G_{N L}=1.00029 G_{L} \text {. }
$$

The value of this correction, $\delta G$, can be evaluated as a difference between 1 and the multiplicative factor of $G_{L}$

$$
\delta G=(1.00029-1) \times 6.6742 \times 10^{-11} \mathrm{~m}^{3} \mathrm{~kg}^{-1} \mathrm{~s}^{-2}=0.19 \times 10^{-13} \mathrm{~m}^{3} \mathrm{~kg}^{-1} \mathrm{~s}^{-2} .
$$

The official error on $G$ is $0.1 \times 10^{-13} \mathrm{~m}^{3} \mathrm{~kg}^{-1} \mathrm{~s}^{-2}$ and therefore the nonlinear correction can be expressed as the double of the official error on $G$. 


\section{Appendix A. The eardrum equation}

The equation

$$
\ddot{x}+x+\epsilon x^{2}=0,
$$

is well known under the name "eardrum equation". It can be solved, see $\frac{14}{\text {, trans- }}$ forming it in

$$
\Omega^{2} \frac{d^{2}}{d T^{2}} X(T)+X(T)+\epsilon(X(T))^{2}=0,
$$

and adopting the method of Poisson that imposes the following solution to $X$

$$
x(T)=x_{0}(T)+x_{1}(T) \epsilon+x_{2}(T) \epsilon^{2},
$$

and to $\Omega$

$$
\Omega=1+\omega_{1} \epsilon+\omega_{2} \epsilon^{2} .
$$

The computer algebra system (CAS) gives

$$
\omega_{1}=0 \quad \omega_{2}:=-5 / 12 .
$$

\section{Acknowledgements}

We thank Richard Enns who has provided us the Maple routine Example 04-S08 extracted from 14 .

\section{References}

1. G. T. Gillies, Reports of Progress in Physics 60 , 151(1997) .

2. P. J. Mohr, B. N. Taylor, Reviews of Modern Physics 77, 1(2005) .

3. P. Baldi, E. G. Campari, G. Casula, S. Focardi, G. Levi, F. Palmonari, Phys. Rev. D $71(2), 022002(2005)$.

4. S. Schlamminger, E. Holzschuh, W. Kündig, F. Nolting, R. E. Pixley, J. Schurr, U. Straumann, Phys. Rev. D $\mathbf{7 4}$ (8), 082001(2006) .

5. J. Schurr, F. Nolting, W. Kündig, Physics Letters A 248 , 295(1998) .

6. C. H. Bagley, G. G. Luther, Physical Review Letters 78 , 3047(1997) .

7. K. Kuroda, Physical Review Letters 75 , 2796(1995) .

8. S. Matsumura, N. Kanda, T. Tomaru, H. Ishizuka, K. Kuroda, Physics Letters A 244 , 4(1998) .

9. J. Luo, D. Wang, Q. Liu , C. Shao, Chinese Phys. Letters 22, 2169(2005) .

10. S. Kononogov, V. Mel'nikov, Measurement Techniques 48, 521(2005) .

11. N. N., Leybold Physics Leaflets Determining the gravitational constant, Leybold, Cologne, 1958.

12. W. H. Press, S. A. Teukolsky, W. T. Vetterling, B. P. Flannery, Numerical recipes in FORTRAN. The art of scientific computing, Cambridge University Press, Cambridge, 1992.

13. J. Luo, D. Wang, Z. Hu , X. Wang, Chinese Phys. Letters 18, 1012(2001) .

14. R. H. Enns, G. C. McGuire, Computer Algebra Recipes for Classical Mechanics, Birkhauser, Boston, 2002. 